\title{
EUROFIT - Integration, Homogenisation and Extension of the Scope of Large 3D Anthropometric Data Pools for Product Development
}

\author{
Rainer TRIEB ${ }^{\mathrm{a}}$, Alfredo BALLESTER ${ }^{\mathrm{b}}$, George KARTSOUNIS ${ }^{\mathrm{c}}$, Sandra ALEMANY ${ }^{\mathrm{b}}$, Jordi URIEL ${ }^{\mathrm{b}}$, \\ Guido HANSEN ${ }^{a}$, Florendia FOURLI ${ }^{c}$, Mirco SANGUINETTI ${ }^{c}$, Michael VAN GENABITH ${ }^{d}$ \\ ${ }^{a}$ Human Solutions $\mathrm{GmbH}$, Kaiserslautern, Germany; \\ ${ }^{\mathrm{b}}$ IBV - Instituto de Biomecánica de Valencia, Spain; \\ ${ }^{\mathrm{c}}$ Hypercliq E.E., Athens, Greece; \\ ${ }^{d}$ Human Solutions of North America, Carey, USA \\ http://dx.doi.org/10.15221/13.258
}

\begin{abstract}
Over the last decades, human body metrics have been used to improve human-product interaction. Along this period, the use of 1D-measurements in "classic" ergonomic applications has been extended to consumer goods industries such as the automotive, apparel, furniture or orthopedic products. New technologies for the gathering, storage and analysis of anthropometric data have boosted the availability of digital anthropometric resources. Since 1999, more than 16 large-scale national 3D body scanning surveys have been conducted around the world (six in Europe). The availability of these data pools has created the opportunity to exploit shape information beyond today's 1D-measurement based use and methodologies. However, these data pools are dispersed and heterogeneous (e.g. different scanning technologies or different protocols) and, above all, the exploitation of 3D data at industry level requires knowledge, skills and resources beyond the means of companies, especially SMEs. These barriers have until now strongly limited the use of existing 3D shape data to scientific and academic research.
\end{abstract}

The paper introduces the EUROFIT project initiative (www.eurofit-project.eu). EUROFIT is a collaborative project co-funded by the European Community which started in June 2012. EUROFIT vision is to unleash the huge potential provided by the increasing number of databases of 3D body scans for the European consumer goods' industries. The project aims to implement an online platform and an open framework that enables designers and industrialists to draw useful 3D shape information and use it in their product development processes in an easy and direct way. R\&D work focusses on the systematization and extension of methods for 3D shape data aggregation and analysis in a reliable but economically sustainable way, as well as on the development of sector-specific applications and user-friendly interfaces.

Keywords: Sizing Surveys, 3D Body Scanning, 3D Surface Anthropometry, Homologous Shape Representation, Shape Data Harmonization, Shape Analysis, iSize

\section{Introduction}

Over the last decades different organizations and consortia have conducted over 30 large-scale national 'sizing surveys' across the world. Each of these scattered digital repositories contains 2.000-20.000 individual datasets. They gather data from more than 250.000 subjects altogether (Figure 1). The number of digital anthropometry data (DAD) repositories thus is rising increasingly since the release of accurate 3D body scanning technologies in the mid of the nineties enabled the efficient collection of digital information on body shape [3]. These data are dissociated from any personal information, but are linked to socio-demographic information such as age, gender or bodyweight. There are two types of DAD repositories (Figure 2):

- 3D body scan databases typically consist of sets of 1-4 dense meshes $(500,000-800,000$ points and 5-40MB each) representing the subject surface in different postures (e.g. sitting, standing).

- 1D measurement databases consist of sets of pre-defined measures of each subject. Recent databases are normally compliant with ISO8559 or ISO7250 [5,6]. Measures can be either obtained manually (callipers and metric tape) or digitally extracted from 3D body scan databases. 


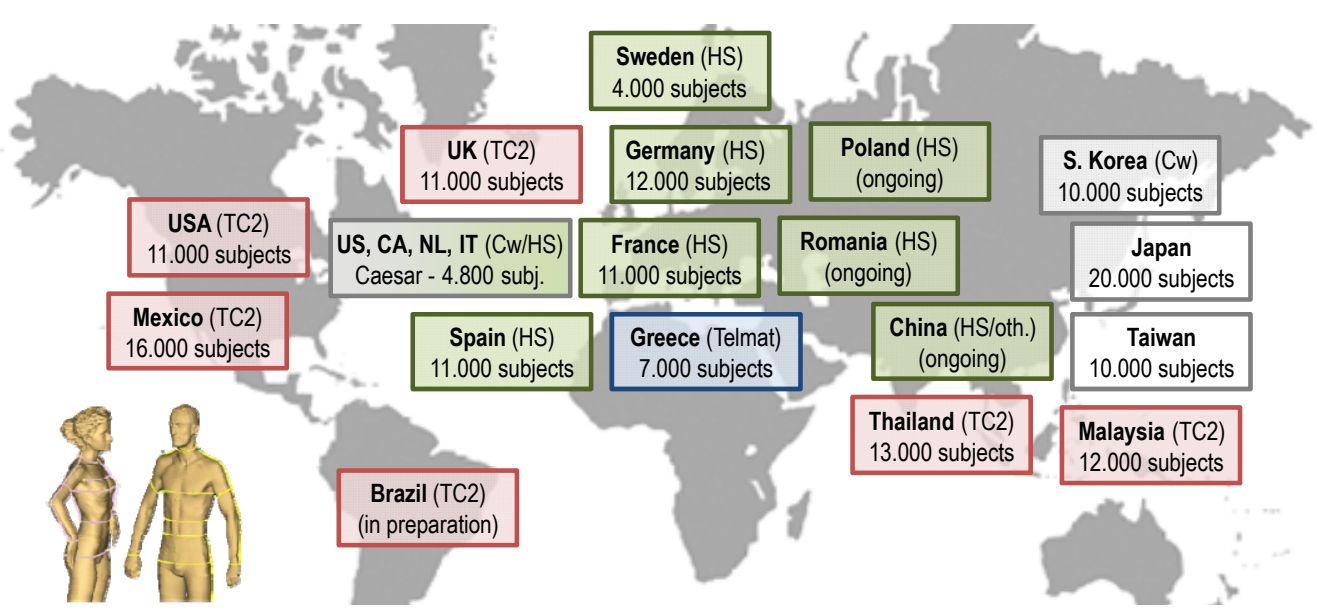

Figure 1: Main 3D scan surveys, showing country (technology) and approx. no. of subjects

The use of human body metrics has become a significant source of product innovation and improvement to industries where consumer fit, comfort and ergonomic considerations are key factors. This is especially the case in fashion (e.g. footwear or apparel), health (e.g. orthotics or prosthetics), transport \& aerospace (e.g. seats or human-machine interfaces), or safety (e.g. protective equipment or workstations) among others. The most widely used anthropometric information comes from aggregated statistics, which often are derived from non-representative or biased population groups (e.g. military). Advanced tools, such as parametric human models or DAD resource portals (e.g. iSize $[1,2]$ - https://portal.i-size.net) improved data access to designers and industrialists. However, all of them, in the best of the cases, are solely based on the analysis of 1D-measures. Although, today huge $3 \mathrm{D}$ information exists only 1D extracts are used because exploiting shape data is not trivial and in most application areas is not accessible to the product developers (Figure 2).

This fact entails important limitations inherent to the use of fragmented 1D-measurement datasets, namely:

- Serious information losses because the 500.000-point meshes are being compressed to less than 100 measures (i.e. lengths and girths). Shape information as base for fit optimization is lost.

- Limited reliability when linked to product development tools (e.g. 2D/3D CAD, CAM) because the use of $1 \mathrm{D}$ data may result in misleading 2D/3D shape interpretations.

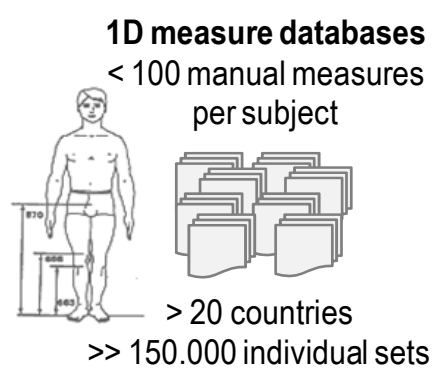

$3 \mathrm{D}$ raw scan databases > 500.000 points per subject

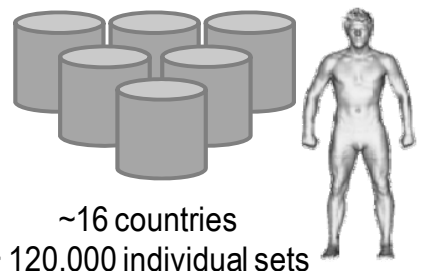

$\sim 120.000$ individual sets

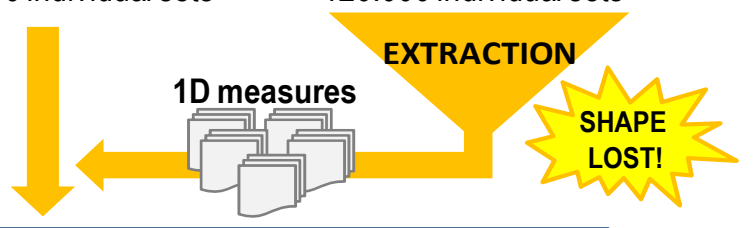

Ready for 1D data analysis and exploitation

Figure 2: Current pooling process leading to $3 D$ shape data fragmentation and loss

- Limited to pre-defined measurement sets because even original application sectors (e.g. apparel ISO8559, or ergonomic design using limb reaches and envelopes ISO7250) may require additional information for optimal designs.

- Limited cross-data analysis of heterogeneous sources, since only similar measurements are comparable [7].

Yet, 3D shape data remains an unexploited resource for industry. Latest research works on SHAPE ANALYSIS [4] have shown the possibility of reliably handling, combining and synthesizing huge amounts of $1 D$ and $3 D$ data in clever ways, to provide potentially useful information for product development: 


\section{The EUROFIT approach and concept}

Enabling reliable and sustainable exploitation of 3D shape information removes the aforementioned limitations of anthropometric data limited to 1D use. It provides industrialists and designers with a new source of product innovation, improvement and differentiation. Further, it revalues existing 3D scan repositories, enabling database owners to obtain a greater value for the data, and incentivising its update and extension. Finally, it allows the creation of novel tools and services reusing the existing digital resources to exploit these into new unforeseen application fields.

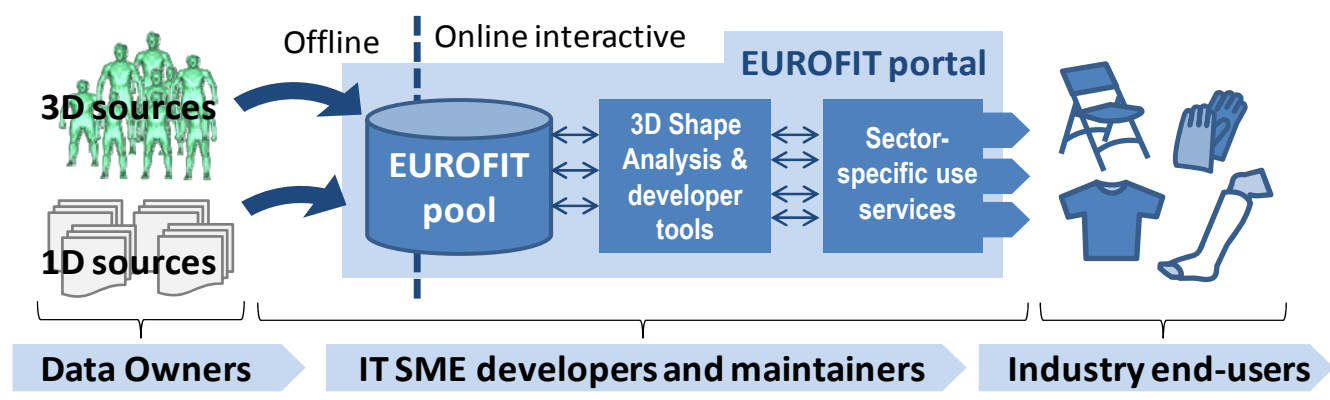

Figure 3: Overview of EUROFIT concept

The EUROFIT concept lies therefore upon the following pillars for an interactive, easy-to-use EUROFIT portal (Figure 3):

- Linking and pooling existing anthropometric resources in a unified 3D anthropometric data portal providing a single point access to international body shape information derived from existing and forthcoming 3D body scan databases as well as from existing 1D-measurement databases.

- Launching dedicated services for the direct use of reliable 3D shape information by industry, providing sector-specific tools to introduce shape information into product design - e.g.; the optimal fit or functional adaptation of product shape; the extraction of new user-defined measures; the definition of statistically representative mannequins with interfaces to export 3D models to virtual prototyping software used in industrial design.

- Creating an open application framework promoting the extension and upgrade of any of the above features by third parties - such as the aggregation of new body part repositories (e.g. head, hand or feet) or the development of new supportive tools for the design of other products.

EUROFIT is a joint co-operation project partially funded by the European Community. It started in June 2012 and will conclude in May 2014. EUROFIT vision is to unleash the huge potential provided by the increasing number of databases of 3D body scans for the European consumer goods' industries (Figure 4). EUROFIT gathers a complementary 6-partner consortium to implement the EUROFIT concept (Table 1). The consortium gathers representatives from 4 Countries (Germany, Greece, Iceland and Spain).

Table 1: EUROFIT project partners

\begin{tabular}{|l|l|l|l|l|}
\hline \multicolumn{1}{|c|}{ Part.no } & \multicolumn{1}{|c|}{ Participant organisation name } & \multicolumn{1}{|c|}{ Short name } & \multicolumn{1}{|c|}{ Country } & \multicolumn{1}{c|}{ Type } \\
\hline 1 (Coord.) & Instituto de Biomecánica de Valencia & IBV & Spain & RTO \\
\hline 2 & Human Solutions GmbH & HS/Assyst & Germany & IT SME \\
\hline 3 & Hypercliq Florendia Fourli \&Co & Hypercliq & 年 Greece & IT SME \\
\hline 4 & Össur hf & Ossur & Iceland & LE end-user \\
\hline 5 & Iturri S.A. & Iturri & Spain & LE end-user \\
\hline 6 & Schrittenloher GmbH & Rieder & Germany & SME end-user \\
\hline
\end{tabular}




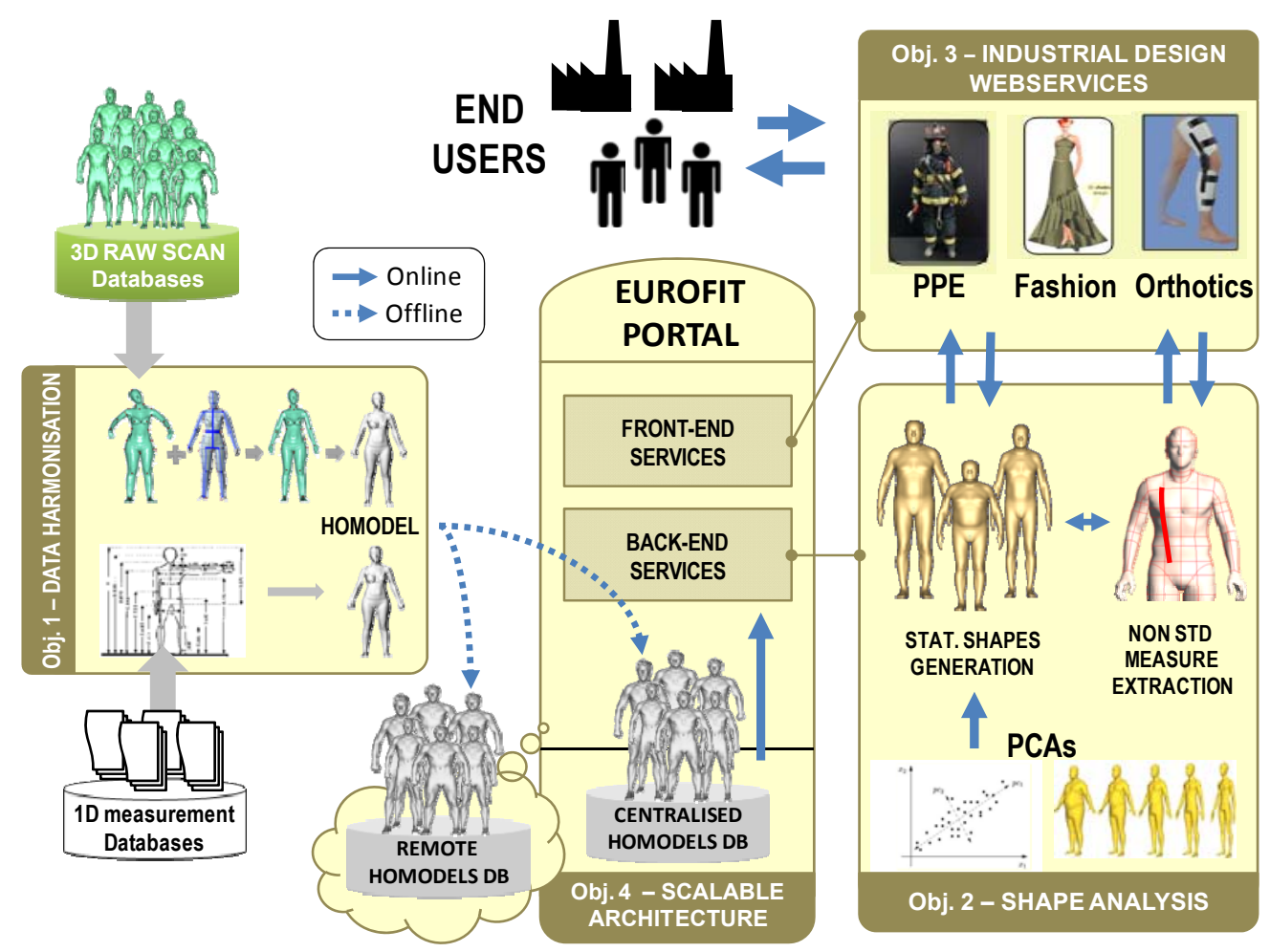

Figure 4: EUROFIT overall diagram illustrating concepts, architecture and S\&T objectives

The project consortium was built in order to guarantee:

- The access to the required background knowledge/technology to be used as a baseline as well as the skills and resources to undertake the challenging RTD proposed work (Human Solutions $\mathrm{GmbH}$, Instituto de Biomecánica de Valencia, Hypercliq EE);

- The access to different databases ,3D and 1D, to both test the developments and aggregate data to launch the EUROFIT portal (Human Solutions $\mathrm{GmbH}$, Instituto de Biomecánica de Valencia, Hypercliq EE);

- The industrial capability to absorb the delivered tools, assure its acceptance and to carry out the demonstration activities (Össur hf - Orthotics, Iturri S.A - Personal Protection Equipment. and Rieder Moden - Fashion); Relevance of end-users in EUROFIT is to ensure that functionalities are market-driven, that early usability testing and development redressing of the portal during development.

Key Scientific and Technical objectives (S\&T objectives) of EUROFIT project are summarized as follows (Figure 4):

1. Maximize the number of databases available to end-users in garment and orthopedics sectors by utilizing both 3D shape data bases and 1D measurement data bases.

2. Create a common 3D homologous mesh structure with body part semantic information able to represent individual body shapes based on 30-70 anatomical references (from hereon called 'homodel').

3. Implement a robust and automated method for creating individual homologous models (homodels) raw scan data coming from different 3D scan technologies.

4. Implement a robust and automated method for the statistical estimation of individual homologous body shapes from sets of existing 1D measurements.

5. Guarantee the usability of the portal by the customers' goods industries, in particular the garment and the orthopedic sectors, by providing use case specific services.

6. Extending the iSize international body measurement data portal to the 3D world and enabling the externalization of the database hosting and data processing through web-services. 


\section{Creation of harmonized 3D-shape databases}

The EUROFIT portal will include body measurements, socio-demographic information (i.e. age, gender, and nationality) and 3D body shape information (i.e. 3D body representation in standing posture plus 30-200 body anatomical references) for analysis. Each database will be made up of these 3 types of data and will be constituted by a representative number of individual datasets. Data in the portal is structured by nationalities according as national measurement or scanning surveys are gathered. Body measurements included in the databases is at present a selection from the 99 measurements currently available at iSize (44 garment construction related measurements, according to ISO 8559 [5], and 55 ergonomics-related measurements, according to ISO 7250 [6]).

\subsection{Homologous shape representation}

3D body shape information in the databases includes a 3D representation of individual data in standing posture following a homologous mesh structure based on anatomical references. This homologous structure has point to point correspondence across the homodels (homologous models) of all the databases included in EUROFIT.

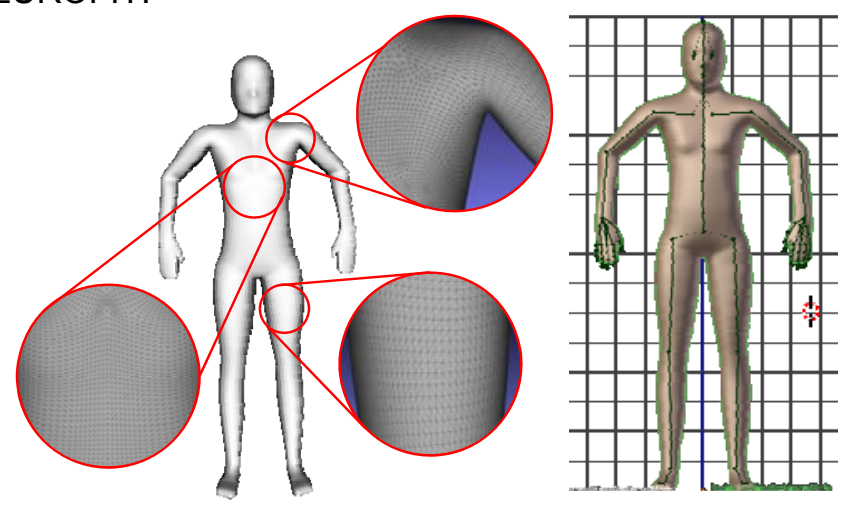

Figure 5: Image of the template mesh topology, geometry and skeleton

EUROFIT has developed a structure constituted by a single closed mesh surface made up of 49.530 vertices and 99.056 triangle faces (Figure 5). Its structure features an high local density providing (1) sufficient resolution for accurate representation of body shapes from 3D raw scan data (i.e. point to point average error below $0.5 \mathrm{~mm}$ ) and (2) light enough to follow multivariate analysis of larger target population selections (i.e. involving from hundreds to thousands individual homodels). This structure enables the association of anatomical references/landmarks to concrete vertices, the segmentation of the mesh into its different semantic parts, and the adoption of a virtual skeleton (Figure 5).

\subsection{Creation of individual homodels from 3D raw scan data}

Creation of homologous representations from 3D scan data specifically with respect to the availability of different postures from various surveys requires a certain degree of standardization. The minimum technical requirements from a database to enable aggregation to EUROFIT are presented in Table 2.

Table 2: Specifications for 3D database aggregation

\begin{tabular}{|c|c|}
\hline $\begin{array}{l}\text { Specifications for } \\
\text { the data } \\
\text { acquisition } \\
\text { protocol }\end{array}$ & $\begin{array}{l}\text { - Scanning posture: Posture A standing defined by EN ISO } 20685 \text { considering that: } \\
\text { - The distance between feet could vary between } 50-600 \mathrm{~mm} \\
\text { - Upper arms abducted to form an angle with the sides of the torso within } 10-70^{\circ} \text {. } \\
\text { - Elbow flexion can range from } 0^{\circ} \text { (full extension) to } 90^{\circ} \text {. } \\
\text { - Scanning attire: Garment defined on EN ISO } 20685 \text { including the following variations. }\end{array}$ \\
\hline $\begin{array}{l}\text { Specifications for } \\
\text { the data input } \\
\text { surface }\end{array}$ & $\begin{array}{l}\text { - Open or closed surface defining a human full body shape in standing posture. } \\
\text { - Any standard or custom format that specifies explicitly or implicitly the xyz coordinates of the } \\
\text { vertices and surface normals at the vertices. } \\
\text { - Minimum number of } 50,000 \text { vertices uniformly distributed: minimum average distance between } \\
\text { neighbours of } 10 \mathrm{~mm} \text {. }\end{array}$ \\
\hline $\begin{array}{l}\text { Additional } \\
\text { information that } \\
\text { must be provided } \\
\text { together with } \\
\text { surface data }\end{array}$ & $\begin{array}{l}\text { - Survey general data: } \\
\text { - Date and locations of the data acquisition } \\
\text { - Minimum socio-demographic data per subject: Age, gender, country } \\
\text { - Minimum complementary anthropometric per subject: Body weight. } \\
\text { - Specifications of the data acquisition system (hardware and surface reconstruction software): } \\
\text { - } \quad \text { technology, xyz resolution, surface reconstruction error }{ }^{1} \text {. } \\
\text { In case presence of physical markers: provide the coordinates of their location }\end{array}$ \\
\hline
\end{tabular}

1 It is recommended to use the standard SO/WD 20685-2 3-D scanning methodologies for internationally compatible anthropometric databases - Part 2: evaluation protocol of surface shape and repeatability of relative landmark positions. 
The creation of the homologous representation from raw scan data consists of two phases: the preprocessing of the raw scan and the homodel creation. In order to enable flexibility in terms scan data input, maximizing the different scanning technologies that could be used as source data, two different convergent processes were followed in order to obtain the homologous representation: template-based method and subdivision-based method. Key advantages/drawbacks from both methods are related to pre-processing steps and input data that are required for following a fully automated homodel creation process.

\subsubsection{D scan data pre-processing}

The pre-treatment process to be applied for locating common valid landmarks enables the direct applicability of template methods to scans obtained from different technologies and surveys. From perceiving $3 \mathrm{D}$ raw data up to the generation of a closed surface mesh representing the body surface geometry a sequence of pre-treatment steps are necessary. This includes e.g. scanning noise reduction, coordinate system \& unit transformation, correction of disoriented face normal, reverse raw data reconstruction, footprint estimation, surface reconstruction, slit modeling, physical marker removal, etc.

Closed surface reconstruction and slit modeling enables reliable landmark identification on the body surface data. The surface reconstruction mechanism comprises generation of a watertight surface representation and closes holes caused by visual occlusions resulting from the sensor configuration or visual occlusions caused by the geometry of the body itself (area under the armpits and in the crotch area). The objective of the slit modeling is to create/recreate reasonable geometries in slit areas (e.g. in the armpits, the crotch), by remodeling these areas in a natural way. Figure 6 exemplarily illustrates an example of the slit modeling of the armpit area after closed surface reconstruction.
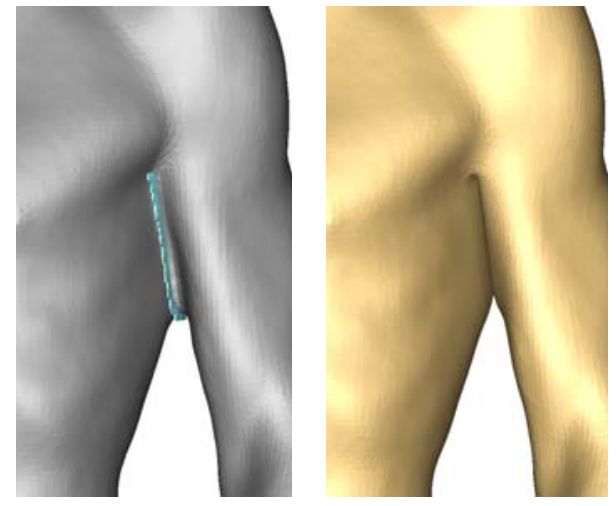

Figure 6: remodeling of slit regions: (a) area under the armpit closed by the previous surface reconstruction, (b) remodeled slit area
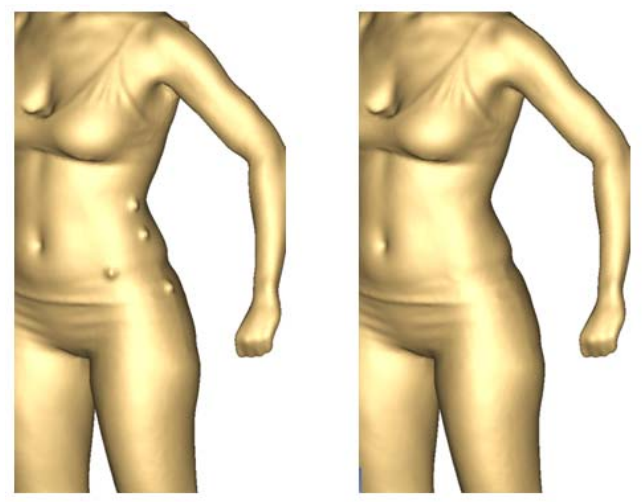

Figure 7: Physical marker elimination (left: scan data with geometry of the markers, right: after automatic elimination)

To support landmark location different methods are commonly used in surface anthropometry surveys: (i) use of additional physical landmarks placed on the subject to create appropriate geometric information in the scan, (ii) interactive identification of landmark locations on the surface scan by a trained user or (iii) automated landmark location for an algorithmically sufficiently well-defined set of predefined landmarks. Specifically in the first case, physical landmarks produce additional geometric arte facts on the scan, which must be eliminated in the pre-treatment process by the physical marker removal (Figure 7).

\subsubsection{Template-based creation of homodels}

Template-based methods are based on fitting a template mesh equipped with a skeleton to the scan using a set of anatomical landmarks as main reference in the fitting process. This method requires that the raw scan has followed several of the aforementioned pre-treatment steps. In particular, after surface reconstruction, hole-filled areas (originally missing information) are identified to be treated differently during the fitting process in an alternative way.

This method consists of three main steps: template posing, template fitting and post-processing. Template posing is necessary to make the template adopt a posture as close as possible to the raw scan. In this process template joints are iteratively moved and bones are iteratively scaled until the 
template adopts a posture and volume that approximates the anatomical references of the template those of the pre-treated scan. Methods developed in the project for the template fitting are adaptations of the combination of the methods presented by Allen et al [8], Amberg et al [9] and Liepa [10]. An image of the result of the fitting process is illustrated in Figure 8.
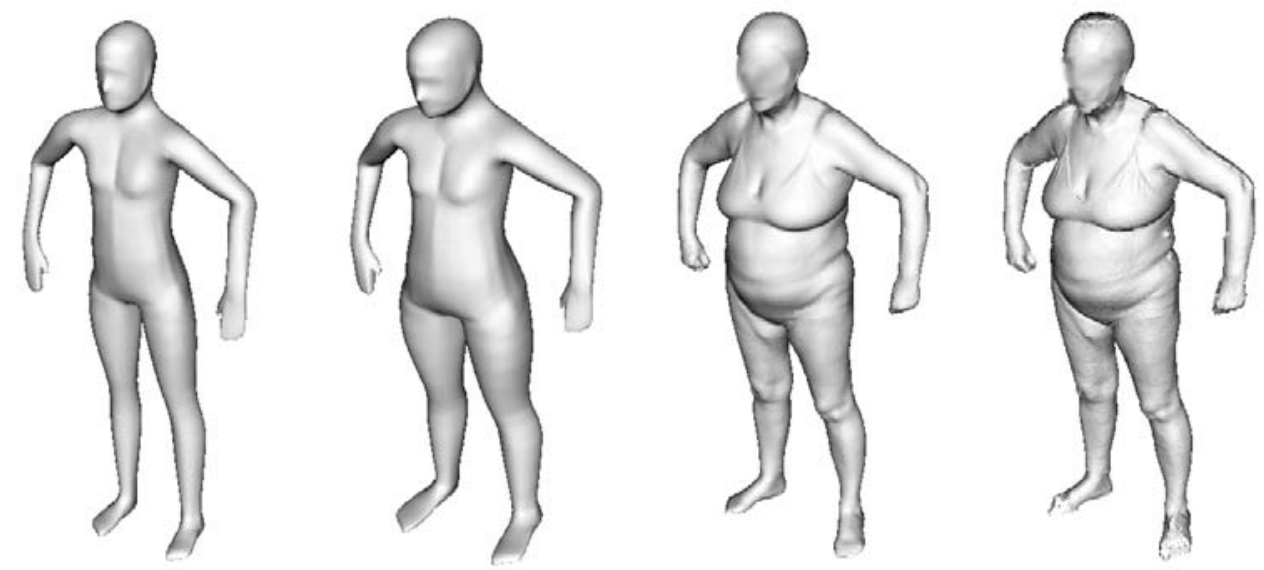

Figure 8: From left to right: Template; Posed template; homodel; Raw scan

At the end of the Template fitting process, information about the quality of the homodel creation is registered at each vertex of the homodel as the distance from the vertex to the target surface of the pre-processed scan. The process developed guarantees an average error below $1 \mathrm{~mm}$ on average on the homodel surface, and near zero in most of the surface. Two sample images of the heat map-like representation of the quality are provided in Figure 9.

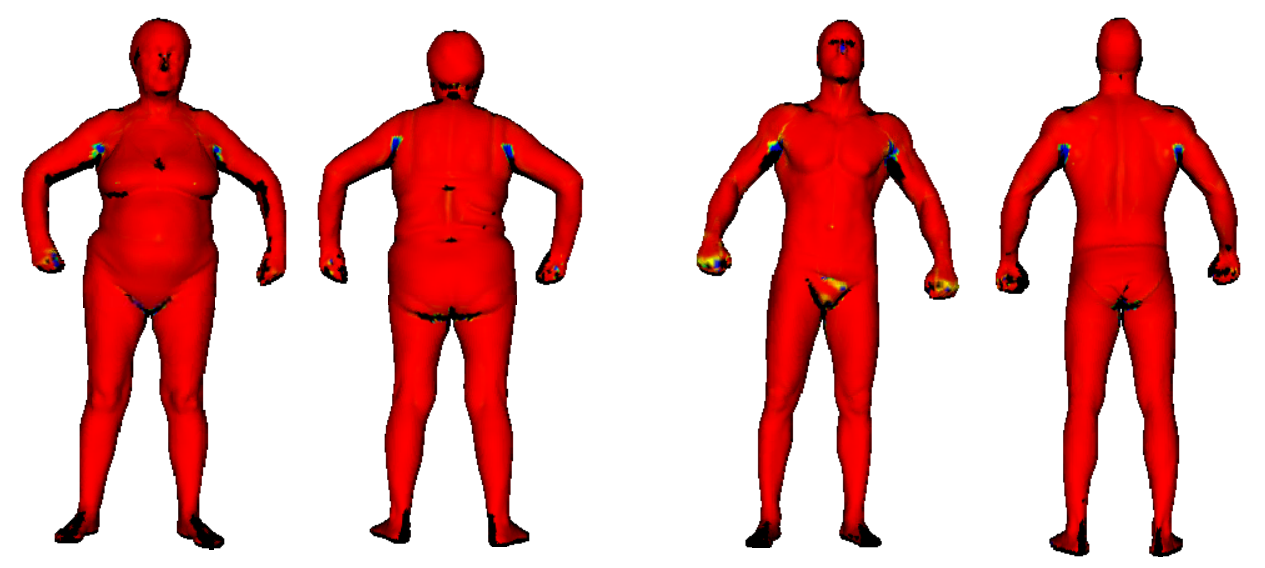

Figure 9: error map in female and male homodels: colour scale ranges from red=0 to blue $\geq 1 \mathrm{~mm}$. Black areas correspond to original holes in the raw scan and have no information about quality

\subsubsection{Subdivision-based creation of homodels}

Subdivision-based method is based on fitting the scan with a coarse mesh defined by anatomical references, which is progressively broken down into a finer mesh that fits the original scan shape. Subdivision-based methods [11] strongly takes into account semantic information of features already extracted on base of the 3D scan data and helps to reduce e.g. the posture adoption problem.

The structure or topology of the mesh is defined by means of this identified semantics on a low-resolution level. In order to achieve the final high-resolution homodel the mesh structure is successively subdivided and the created new vertexes are adapted to the actual geometrical shape of the individual 3D scan. Figure 10 illustrates the mesh creation and mesh refinement process.

The advantage of the method is to create homodel structures in different resolutions and refinements. In order to achieve structural compliance with the EUROFIT template mesh structure a conversion method between different homodel topologies originating from different sources has been developed. This further allows e.g. to overcome limitations concerning dimensionality (i.e. the resolution or number of triangles) of the homodels with respect to computability (e.g. PCA analysis) in a thorough way. 


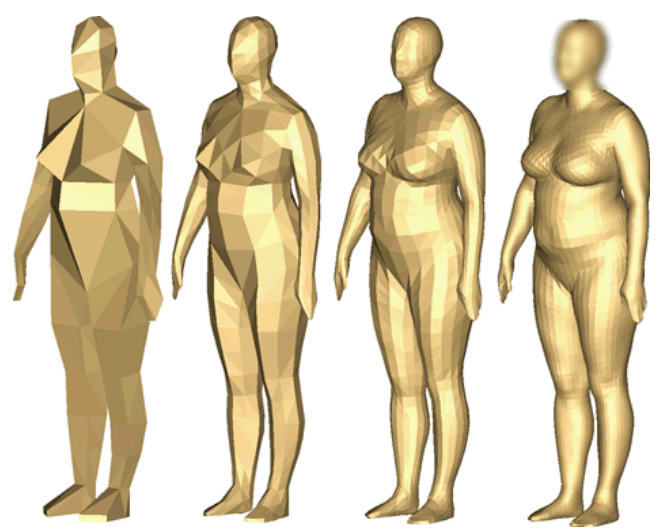

Figure 10: Subdivision-based homodel creation: Starting from a low resolution mesh adjusted to extracted and identified landmarks, the mesh is subdivided and refined gradually to the individual $3 D$ body shape.

\subsection{Estimation of homodels from individual measurements}

Most of the anthropometric databases available consist of collections of individual measurement sets. Since one of the main aims of EUROFIT is to maximize the number of databases available for 1D and 3D analyses a method for re-creating individual statistical body shapes from individual measurement sets was implemented. This method is enabled by the availability of a homologous model database.

The reconstruction is achieved in two steps: body shape approximation and body shape refinement. Body shape approximation is based on an adaptation of Allen et al [12] using data collected from 3D body scans and corresponding 1D measurements for creation of a reference database To solve the recreation problem we use Principal Components Analysis (PCA) to reduce data dimensionality. Body shape refinement is based on iterative measurement and search of the desired measurements. It is achieved by the application of a minimization method using gradient-descent methods [13] where the variables are the PCA scores minimizing the deviations from the desired measurements. In order to illustrate the results obtained by this method the 20 measurements of several women from the Spanish database that did not take part in 500 training set were used.

Figure 11 shows the differences in the measurements obtained compared to the original sample women. As it can be observed, the differences in measurements after the approximation are below 2 $\mathrm{cm}$ and after the refinement are close to zero. Moreover physical resemblance of the body areas with measurements involved (i.e. trunk and legs) is very high.

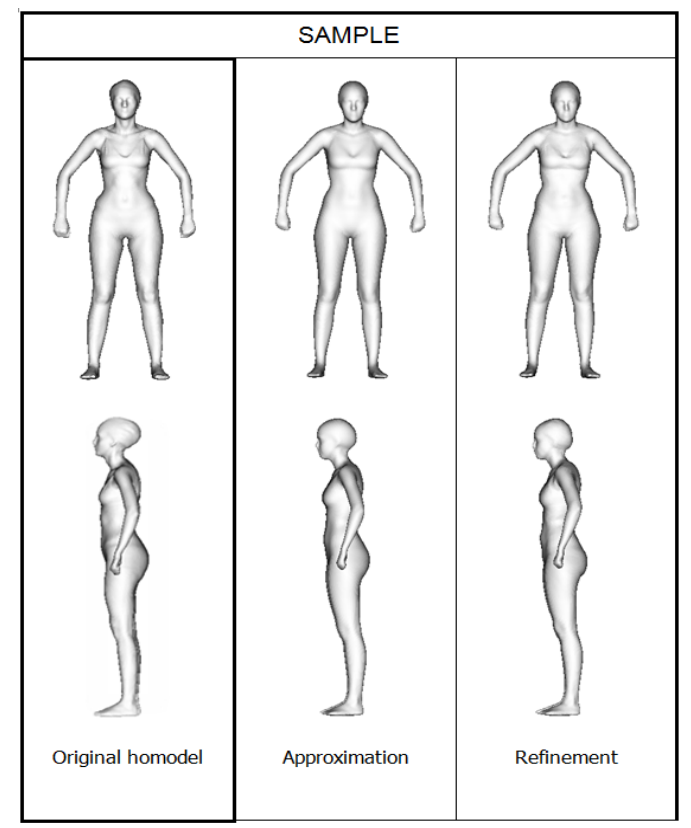

\begin{tabular}{|l|c|c|c|}
\cline { 2 - 4 } \multicolumn{1}{c|}{} & \multicolumn{3}{c|}{ SAMPLE 1 } \\
\cline { 2 - 4 } \multicolumn{1}{c|}{} & Original & Approx. & Refinement \\
\hline Body height & 1737.76 & 1740.94 & 1737.76 \\
\hline Neck height & 1499.9 & 1501.43 & 1499.9 \\
\hline Waist height & 1062.17 & 1064.71 & 1062.17 \\
\hline Buttock height & 881.571 & 883.742 & 881.571 \\
\hline Hip height & 924.869 & 929.999 & 924.868 \\
\hline Crotch height & 823.341 & 827.068 & 823.341 \\
\hline Knee height & 484.844 & 485.106 & 484.844 \\
\hline Ankle height & 80.2985 & 81.4742 & 80.2986 \\
\hline Breast height & 1274.24 & 1275.53 & 1274.24 \\
\hline Bust girth & 842.947 & 846.31 & 842.947 \\
\hline Underbust girth & 702.44 & 700.995 & 702.44 \\
\hline Waist girth & 643.892 & 644.247 & 643.892 \\
\hline Hip girth & 1026.54 & 1027.06 & 1026.54 \\
\hline Max. belly girth & 802.47 & 801.244 & 802.47 \\
\hline Thigh girth left & 581.447 & 569.718 & 581.447 \\
\hline Thigh girth right & 568.359 & 567.096 & 568.359 \\
\hline Knee girth left & 363.816 & 370.576 & 363.816 \\
\hline Knee girth right & 380.05 & 372.231 & 380.05 \\
\hline Calf girth left & 348.519 & 351.18 & 348.519 \\
\hline Calf girth right & 349.12 & 349.689 & 349.12 \\
\hline
\end{tabular}

Figure 11: a) sample of the recreation of 3D shapes from measurement sets, b) measurement comparison of the original samples with the re-created shapes (in $\mathrm{mm}$ ) 
The minimum requirements for the aggregation of measurement databases to EUROFIT portal are described in Table 2.

Table 2: Specifications for measurement database aggregation

\begin{tabular}{|c|cc|}
\hline $\begin{array}{c}\text { specifications for } \\
\text { the }\end{array}$ & $\begin{array}{c}\text { A collection of at least } 20 \text { anthropometric variables including stature and weight characterizing } \\
\text { the size (lengths and girths) of the main body segments (i.e. head, neck, shoulders, forearm, } \\
\text { upper arm, torso, thigh, calf) and joints. }\end{array}$ \\
\hline $\begin{array}{c}\text { Additional } \\
\text { measurements }\end{array}$ & $\begin{array}{l}\text { Thescription of the anthropometric variables, including the body posture and the anatomical } \\
\text { points reference. }\end{array}$ \\
$\begin{array}{c}\text { information that } \\
\text { must be }\end{array}$ & $\begin{array}{l}\text { Survey general data: } \\
\text { provided }\end{array}$ & $\begin{array}{l}\text { Date and locations of the survey } \\
\text { Sample size and socio-demographic stratification. }\end{array}$ \\
together with & - $\begin{array}{l}\text { Minimum socio-demographic data per subject: Age, gender, country/town } \\
\text { surface data }\end{array}$ & - $\begin{array}{l}\text { Minimum complementary anthropometric per subject: Body weight. } \\
\text { Specifications of the measurement instruments used (either manual or digital) }\end{array}$ \\
\hline
\end{tabular}

\section{EUROFIT portal beyond iSize portal}

The EUROFIT portal (Figure 12) is built upon the commercial iSize portal and used as the experimental and prototyping platform for the integration of 3D shape functionality. iSize is at the same time the most comprehensive single repository of statistically representative 1D measurements that offers 1D interactive analysis for apparel. Sector-specific tools target three consumer product industries, dominated by SMEs, where reliable information about body shape can bring great benefits, stimulating innovations based on the improvement of product performance. In particular, Orthopedic products and Personal Protective Equipment (PPE) are functional products hence performance can be severely affected by inadequate fit or discomfort. Fit is essential to Fashion apparel, which will facilitate use of iSize services and rapidly aggregate demand by end-users.

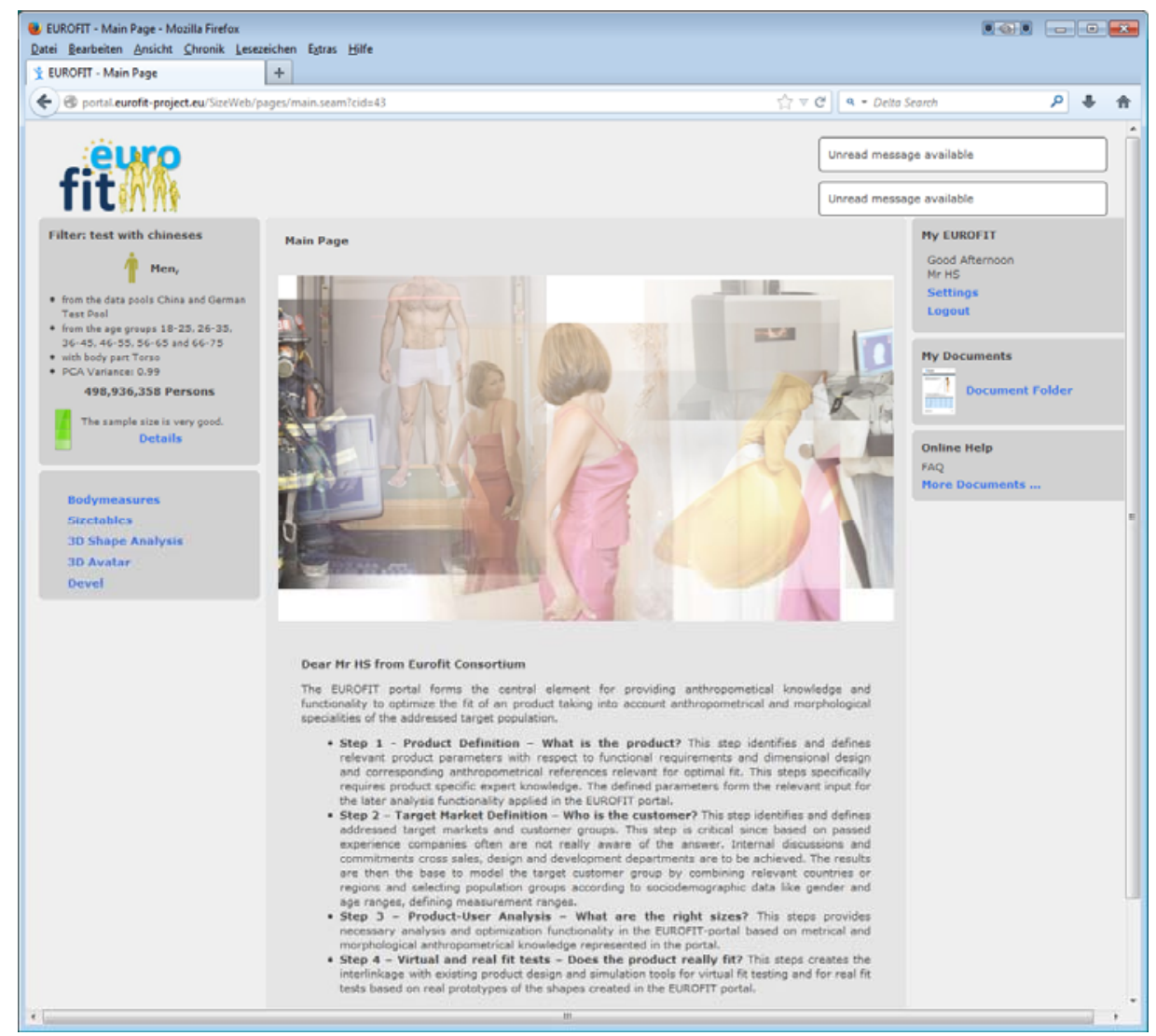

Figure 12: Start screen of the EUROFIT portal 


\subsection{Decentralization of data and services}

The EUROFIT-portal enables its upgrade and up-scale to new data and services adapted to the needs of data owners, developers and end-users. The main challenges related to its implementation were: (1) secure hosting of centralized and decentralized databases of homologous models, (2) enabling further development and integration of application-specific services to exploit the new 3D DAD resources, by defining and providing means and interface framework enabling innovative firms to create novel data analysis tools and services, or recombine the existing; and (3) reusing of existing 1D-based analyses functionality and to combined it with the new 3D analysis functionality and services. Thus, one basic approach for the EUROFIT portal architecture was to create a distributed system architecture and open framework development for a service oriented, distributed architecture implementation.

\subsubsection{Decentralization of external data analysis services}

In this scenario the data of a survey has been pre-processed and integrated to the central server. The $3 \mathrm{D}$ data is being represented in the central homodel data base. Functionality necessary (e.g. PCA exploration of the selected target group) is available on an external server. The principle process is shown in Figure 13:

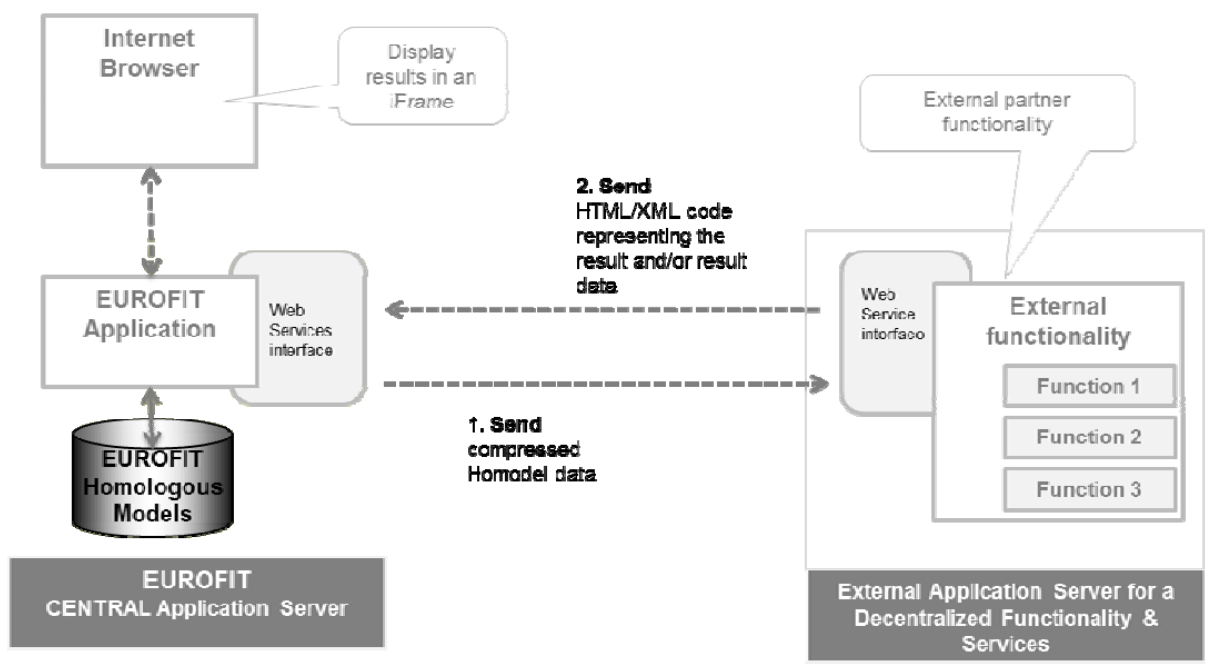

Figure 13: Access to functionality on a national server

In this scenario two approaches can be distinguished. In one case the external functionality delivers back a data object (e.g. a generated shape) for which the graphical representation then has to be handled by the EUROFIT server itself. In the second case, the external server provides its own GUI displayed in an Inline Frame in the EUROFIT end-user browser window. Further control flow is completely transferred to the external service and thus can provide inline is own control elements.

\subsubsection{Decentralization of database hosting}

External analysis functionality requires transfer of data from the central server to the service provider and data volumes transferred between distributed functional units are critical to the performance of the overall system design. 3D shape information is the primary data to be transferred within EUROFIT between the portal centrally hosting the population sample and an external service providing analysis functionality or vice versa.

The following table shows achievable compression rates based on the assumption, that a subset of 1000 individuals has been selected for an analysis to be applied to this data. In case this data has to be transferred the resulting volumes in different representations have been calculated, assuming (i) a scan represented by approx. 0.5 Mio. 3D points and (ii) the resulting data volume based on the homologous representation with approx. 50.000 triangles.

Figure 14 illustrates, that a functionally distributed approach for the original scan data would result in a required data transfer volume of approx. 6 GB of data for a sample of 1000 subjects. Even in the case of transferring the homologous based representation this would result in a volume of $600 \mathrm{MB}$ (Figure 14). 


\begin{tabular}{|c|c|c|c|c|c|}
\hline $\begin{array}{l}\text { Data volumes of } \\
\text { 3D shape data representations }\end{array}$ & 3D Scan & Homodel & $\begin{array}{c}\text { PCA } \\
\text { Compress } 1\end{array}$ & $\begin{array}{c}\text { PCA } \\
\text { Compress } 2\end{array}$ & $\begin{array}{c}\text { PCA } \\
\text { Compress } 3\end{array}$ \\
\hline \multicolumn{6}{|l|}{ 3D Scan based representation of an individual } \\
\hline - Data points per individual & 500.000 & 50.000 & & & \\
\hline - Data size per point (Bytes) & 12 & 12 & & & \\
\hline - Data size per individual (kBytes) & 6.000 & 600 & & & \\
\hline \multicolumn{6}{|l|}{ PCA based representation of an individual } \\
\hline - Number of used eigenshapes (modes) & & & 100 & 50 & 20 \\
\hline - Data size of eigenshapes (kBytes) & & & 60.000 & 30.000 & 12.000 \\
\hline - Score values per individual & & & 100 & 50 & 20 \\
\hline - Data size score values per individual (Bytes) & & & 400 & 200 & 80 \\
\hline \multicolumn{6}{|l|}{ Representation of a target group } \\
\hline - Size of selected target group & 1.000 & 1.000 & 1.000 & 1.000 & 1.000 \\
\hline - Total data size (MBytes) & 6.000 & 600 & 60,4 & 30,2 & 12,08 \\
\hline Compression rates & $100 \%$ & $10 \%$ & $1,01 \%$ & $0,50 \%$ & $0,20 \%$ \\
\hline
\end{tabular}

Figure 14: Comparison of data volumes reached by PCA based data compression

By applying the PCA based compression transferred data volumes can strongly be reduced depending on the degree of accuracy required as basis for the data analysis. In case of 100 eigenshapes the resulting data volume is approx. $60 \mathrm{MB}$ (approx. $1 \%$ of the original data volume), for 50 eigenshapes this volumes is halved and for a 20 eigenshapes representation, the volume reduces to approx. $12 \mathrm{MB}$ representing a percentage of $0.2 \%$ of the original volume, i.e. a compression rate by a factor of 500 is achieved.

\subsection{Data analysis features}

In order to analyze and use shape information from large samples of 3D homodels describing human body shape, complex analyses must be applied. Mesh deformation and principal component analysis (PCA) are the most common methods used in 3D body shape analysis. PCA is the most suitable since it provides with the statistical control required for applying results to engineering and design problems. PCA is a statistics-based method that enables to synthesize smartly high volumes of information [14, $15,16]$.

\subsubsection{Shape data analyses}

Methods for the conduction of fast online PCA were developed and optimized in the project as well as initial interfaces for the exploration (i.e. interactive navigation) and exploitation of combined 3D and 1D data (e.g. correlations of measurements with the principal components to support visual information).

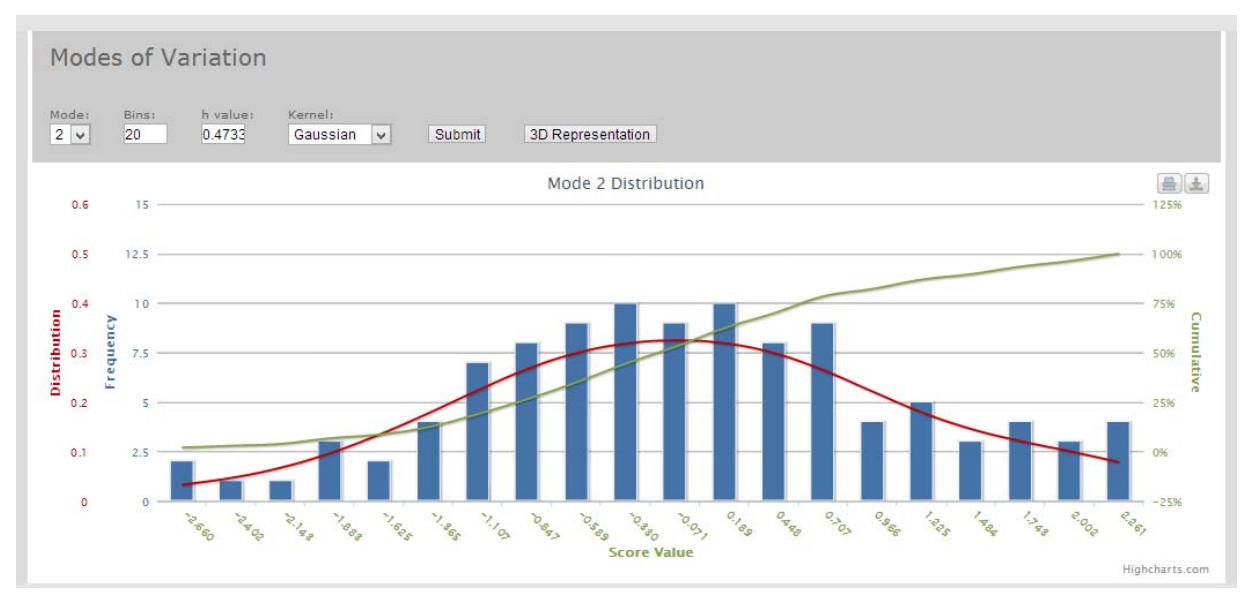

Figure 15: Histogram displaying the distribution of score values for the 1 st mode of variation (Screenshot from the developed application) 
Modes of Variation: Various 2D plots and 3D representations facilitating the understanding of the meaning/interpretation of each mode of statistical variation (principal component or eigenvector) in terms of the distribution of the related PC scores (the values that each mode of variation takes across the selected sample of 3D bodies or body parts). Figure 15 illustrates examples of the prototype tools developed.

Data Visualization: Three dimensional visualizations of selected body parts with statistical significance, such as e.g. 3D representation of the shape pertaining to the 5th percentile of the distribution of values of the given mode of variation or of combined modes of variation (PCs) help to understand the notion of the modes of variations. Figure 16 illustrates an example.

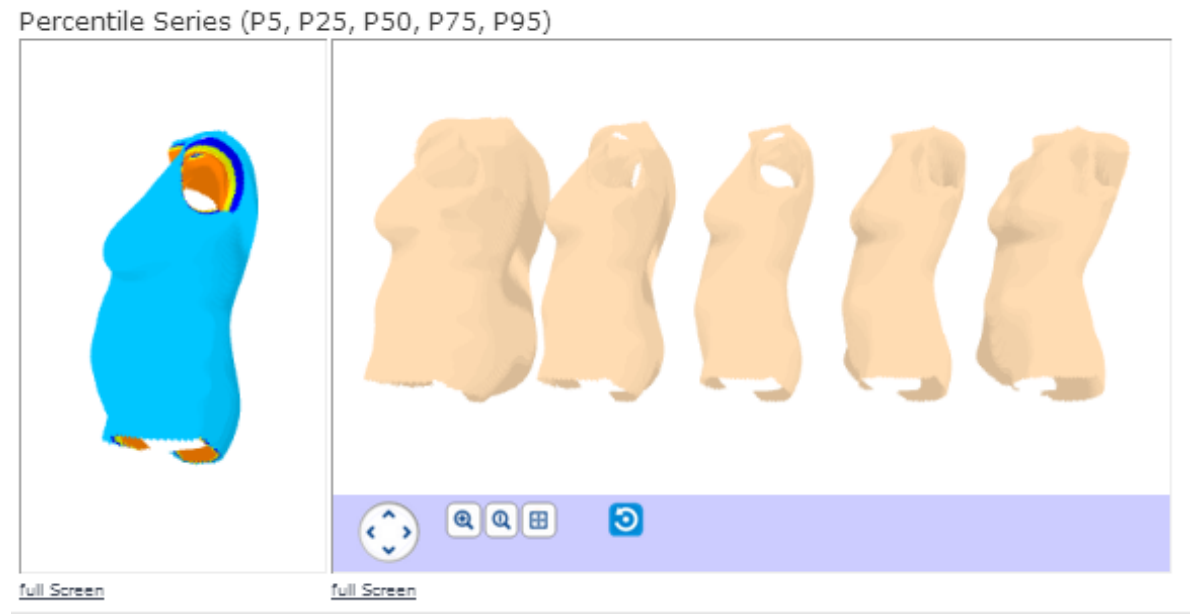

Figure 16: Screenshot of the web-service prototype implemented for the $3 D$ navigable visualization of principal components for a female torso display different "shape"-percentiles

\subsubsection{D-enabled filters for shape data analyses}

The Homodel posture may have an influence when conducting shape analysis of homodel data when using techniques such as PCA. This influence becomes more important if the analysis is conducted over a sample that includes homodels originating from different surveys or data pools, which scans were taken in slightly different standing postures. During shape analyses, different approaches can be followed in order to eliminate, reduce or mitigate the abovementioned influence in an automated or systematic way. The approaches that followed in the project to complement basic shape analyses based on PCA were: conducting automated slight posture corrections and segmenting body parts.

Homologous structure enables to make vertex or face selections by ID in a very simple way, and thus select the same region for a sample of homodels. Focusing body parts of interest for the product prior to the analysis, in order to eliminate posture noise caused by body parts, which are not directly related to the product to be developed.

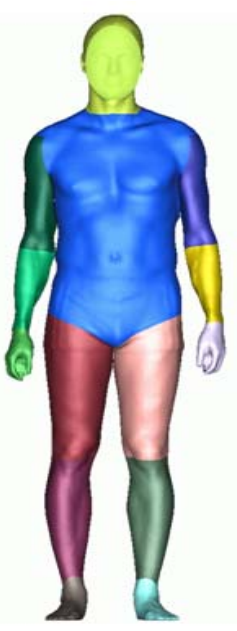

Figure 17: Body part selection segments
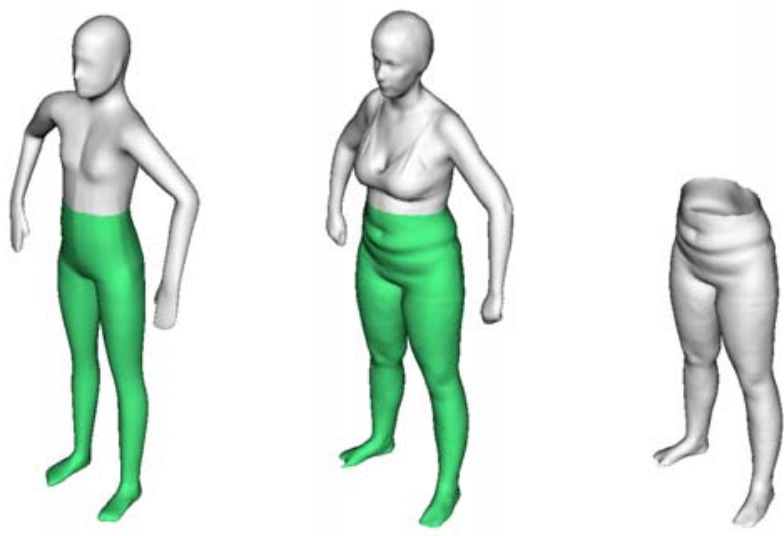

Figure 18: Example of custom selection made for trousers product analysis. From left to right: highlighted trousers selection in the template mesh; highlighted trousers selection in a homodel of the database; and trousers selection in a homodel 
To this end two alternative strategies were followed. On the one hand a pre-defined body segmentation was implemented by dividing the homodel structure into 14 semantic body parts (Figure 17). This strategy enables the user to select the body parts that are related to their product. Second strategy is to enable user-defined custom selections for special products that cannot be made by list selection (Figure 18).

\subsubsection{Creation of statistical avatars / virtual mannequins}

Creation of statistically valid avatars (e.g. average shape of a population group) is one of the main interests for the development of consumer goods products. While shape analyses methods can help product managers and designers to determine which shapes should be used as avatars representing for instance sizes, a parametric tool for the generation of statistical shapes was developed to create them using as input the body measurements (Figure 19).

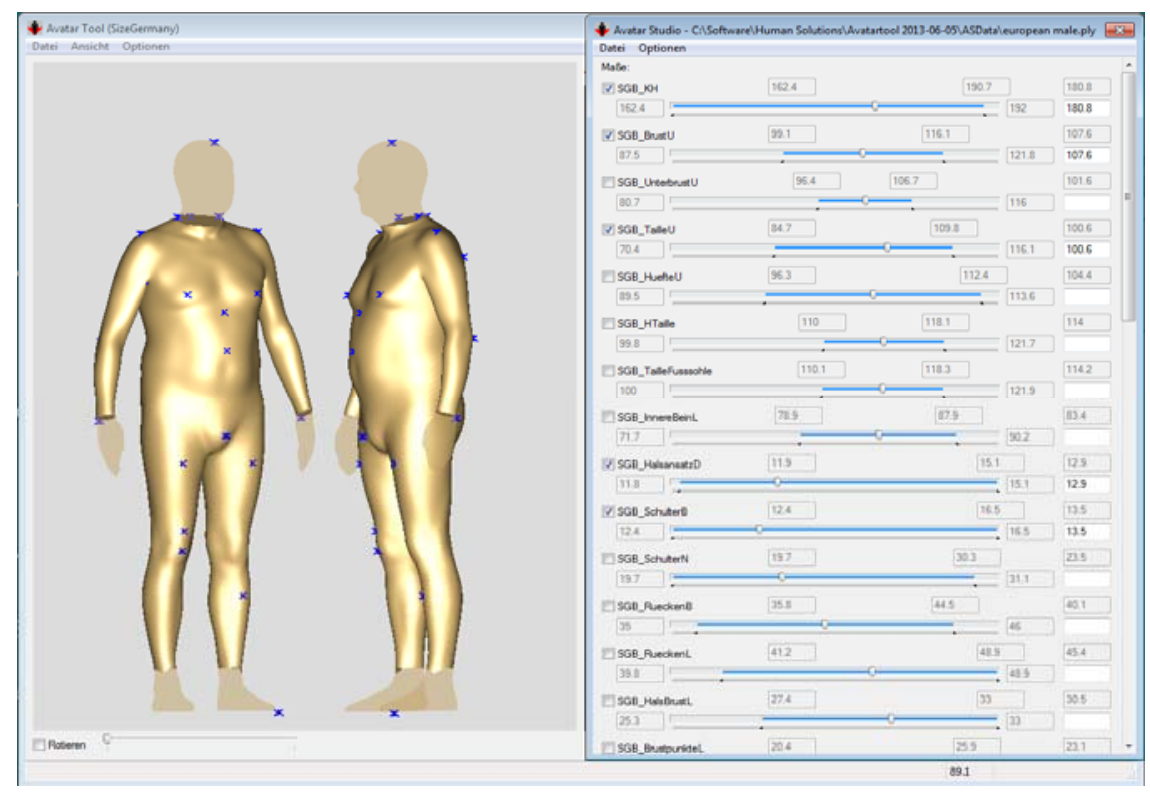

Figure 19: Screenshot of the interactive avatar generation tool: sliders allow the end-user to adjust body measurements for creation of statistically valid avatars

\section{Conclusions and future work}

The EUROFIT project follows a unified approach for handling and analysis of anthropometrical survey data originating from both, 3D surface anthropometry and 1D body measurement based data bases.

In the next project phase the appropriateness of the approach will be validated in cooperation with the industrial end-users integrated in the EUROFIT project. The use-cases include development of optimized sizing systems to specific target groups for PPE (personal protection equipment), 3D shape based fit optimization for orthotic products as well as optimized sizing systems for both off-the-rack garments and made-to-measure garments. The activities will mainly focus on user trials with the databases currently integrated and usability and possible extension and optimization of the developed functionality.

The developed and tools are foreseen to become commercially available in autumn next year and will enable designers and industrialists to draw useful 3D shape information and use it in their product development processes in an easy and direct way without loosing reference to their previous work mainly based on one-dimensional body measurement data.

The research leading to these results has received funding from the European Union Seventh Framework Programme FP7/2007-2013 - ICT Information and Communication Technologies - under grant agreement $n^{\circ} 296116-$ EUROFIT. 


\section{References}

1. iSize - The international measurement and sizing portal iSize, https://portal.i-size.net.

2. Rissiek, A.; Trieb, R. (2010): "iSize - Implementation of international anthropometric survey results for worldwide sizing and fit optimization in the apparel industry", Int. Conference on 3D Body Scanning Technologies, Lugano, Switzerland, 19-20 Oct. 2010, pp. 269-281.

3. Trieb, R.; Seidl, A.; Rissiek, A; Wirsching, H.-J.(2008): "Weltweite anthropometrische Reihenmessungen mit BodyScanning - Überblick über die wichtigsten Projekte, Verfahren und Ergebnisse“, 54. GfA Frühjahrskongress, 9.-11. April 2008, München.

4. C.Shu,(2010):"Making 3-D Anthropometric Data Usable", International World Engineering Anthropometry Resource Conference, 2010.

5. ISO 8559, (1989): ISO International Standard on "Garment construction and anthropometric surveys-Body dimensions".

6. ISO 7250-1, (2008). "Basic human body measurements for technological design" - Part 1: Body measurement definitions and landmarks.

7. Trieb, R.; Seidl, A.; Gordon, C. ; Bradtmiller, B.; Corner, B. D.; Paquette, S. P. (2009): "Comparability of 3D Scan Extracted Measurements and Traditional Measurements - A practical application of the ISO 20685 Standard", IEA 2009, Bejing.

8. Allen, B., Curless, B., \& Popović, Z. (2003): "The space of human body shapes: reconstruction and parameterization from range scans". In ACM Transactions on Graphics (TOG) (Vol. 22, No. 3, pp. 587-594). ACM.

9. Amberg, B., Romdhani, S., \& Vetter, T. (2007): "Optimal step nonrigid icp algorithms for surface registration". In Computer Vision and Pattern Recognition, 2007. CVPR'07. IEEE Conference on (pp. 1-8). IEEE.

10. Liepa, P. (2003): "Filling holes in meshes". In Proceedings of the 2003 Eurographics/ACM SIGGRAPH symposium on Geometry processing (pp. 200-205). Eurographics Association.

11. Praun, E., Sweldens, W., \& Schröder, P. (2001): "Consistent mesh parameterizations”, in Proceedings of the 28th annual conference on Computer graphics and interactive techniques (pp. 179-184). ACM.

12. Allen, B., Curless, B., \& Popovic, Z., (2004): "Exploring the space of human body shapes: Data-driven synthesis under anthropometric control". SAE Digital Human Modeling, 01-2188.

13. Zhu, C., Byrd, R., Nocedal, J., Morales, J. L.:" L-BFGS-B".

14. Allen, B., Curless, B., \& Popović, Z. (2003): "The space of human body shapes: reconstruction and parameterization from range scans". In ACM Transactions on Graphics (TOG) (Vol. 22, No. 3, pp. 587-594). ACM.

15. Ben Azouz, Z et al. (2005): "Extracting Main Modes of Human Shape Variation from 3-D Anthropometric Data", 5th IC3D, 2005.

16. Chang Shu, et al. (2011): "Geometric and Statistical Methods for Processing 3D Anthropometric Data", Int. Symposium on Digital Human Models, 2011.

17. Baran, Ilya; Popovic, Jovan (2007): "Automatic Rigging and Animation of 3D Characters", SIGGRAPH 2007, http://www.mit.edu/ ibaran/autorig/. 